\title{
El empleo público en Castilla y León: una visión comparada 1987-1998
}

\section{Juan Carlos Rodríguez Caballero * y Asunción Valiente García *†}

\author{
En recuerdo de Asun
}

\section{Introducción}

La peculiar evolución de las principales magnitudes del mercado de trabajo (actividad, ocupación, paro, salarios, contratación temporal, etc.) en el pasado inmediato de nuestro país ha llevado a multitud de investigadores a intentar explicar, empleando enfoques y técnicas muy diversas, su comportamiento. En este sentido, la Comunidad de Castilla y León presenta varias regularidades de interés, asociadas tanto a la actividad como a la ocupación, objeto de recientes investigaciones.

Sin embargo, el papel que las Administraciones Públicas han jugado como promotoras directas o indirectas de puestos de trabajo no ha sido suficientemente tratado a escala nacional y obviado, casi de forma absoluta, en Castilla y León ${ }^{1}$.

Este artículo examina el empleo público en la economía de Castilla y León entre 1987 y 1998, cubriéndose así más de un ciclo completo de contracción y expansión del empleo. El trabajo tiene un doble objetivo: en primer lugar, cuantificar el peso relativo del empleo público en Castilla y León y caracterizar a los trabajadores públicos de Castilla y León considerando tanto ciertas características personales, como la edad, el sexo y el nivel de estudios, como características propias del puesto de trabajo; en segundo lugar, comparar, desde las dos perspectivas señaladas anteriormente, el empleo público de Castilla y León con el empleo público de España en su conjunto.

Aunque se trata de un estudio esencialmente descriptivo, la mera constatación de los hechos más sobresalientes nos dará una idea de hasta qué punto ha sido relevante el papel del empleo público en el comportamiento reciente del mercado de trabajo de Castilla y León y nos permitirá señalar los rasgos diferenciales que la evolución y composición de esta variable presenta con respecto al conjunto de España.

La fuente estadística utilizada para la confección del trabajo ha sido la Encuesta de Población Activa (EPA), elaborada por el Instituto Nacional de Estadística (INE). Se trata de la fuente disponible más completa para el estudio de las magnitudes laborales. No obstante, para acometer en profundidad el estudio del fenómeno que nos concierne a escala regional, la información que periódicamente publica el INE resulta insuficiente. Por ello las series de datos se han obtenido mediante petición directa a dicho organismo. Dichas series detallan para España, Castilla y León y sus provincias y desde el segundo trimestre de 1987 hasta el segundo trimestre de 1998, el número de asalariados según su pertenencia al sector público o privado de la economía, y según el tipo de Administración Pública en la que trabajan ${ }^{2}$.

Además, para ahondar en las características del empleo público (tales como el nivel de formación académica, la educación complementaria, el nivel socioeconómico, etc.) se ha recurrido a los microdatos de la Encuesta de Población Activa, esto es, a las respuestas individuales que cada uno de los sujetos entrevistados da a las distintas preguntas de la encuesta. Dado el volumen de datos que conlleva este nivel de desagregación, se ha solicitado la misma para dos momentos puntuales: el segundo trimestre de 1987 y el segundo trimestre de 1998, de forma que puedan efectuarse comparaciones entre los dos años extremos del período de tiempo considerado en el análisis. 
El trabajo se estructura de la siguiente manera: primeramente, tras este apartado introductorio, se analiza la evolución del peso relativo de los asalariados públicos de Castilla y León respecto al total de asalariados de la economía regional. A continuación, en el apartado tercero se analiza la distribución del empleo público de Castilla y León por sectores de la Administración Pública así como la variación producida en dicha distribución entre el inicio y el final del período considerado. Posteriormente, en el apartado cuarto, se presenta una caracterización de los empleados públicos castellano-leoneses. Finalmente, en el último apartado, se recogen los rasgos más sobresalientes en relación con la estructura del empleo público en Castilla y León.

\section{Evolución del peso relativo del empleo público en Castilla y León}

La Tabla 2.1 indica el número de asalariados totales, del sector privado y del sector público de la economía española y de Castilla y León.

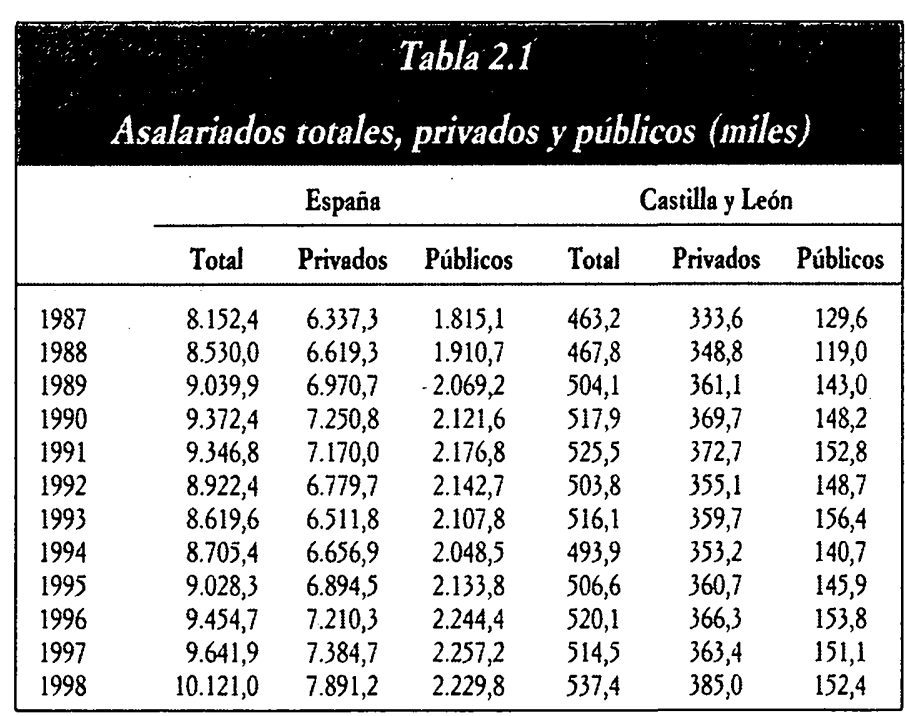

Fuente: Elaborada a partir de los datos de la EPA.

El primer rasgo que la información estadística pone de manifiesto es que el peso relativo de los asalariados públicos sobre el total de asalariados se sitúa en Castilla y León por encima del peso relativo que tienen en el conjunto del país en más de cinco puntos porcentuales por término medio (Gráfico 2.1). Esta diferencia, aunque de menor cuantía, se mantiene si consideramos el peso relativo que el sector público (servicios no destinados a la venta) tiene en el valor añadido total que se genera en cada uno de los dos ámbitos espaciales ; entre 1987 y 1996, el peso del sector público en la economía castellano-leonesa es superior en 3.3 puntos porcentuales por término medio al peso que el sector público tiene en el conjunto del país (Gráfico 2.2).

En el período considerado se ha producido un leve aumento del peso relativo de los asalariados públicos de Castilla y León. En 1987 el 28,0\% del total de asalariados de Castilla y León eran públicos mientras que en 1998 el porcentaje se sitúa en el $28,4 \%$. En la economía nacional se ha mantenido el peso relativo de los asalariados públicos, situándose en ambos años en el $22 \%$.

Tanto en el ámbito regional como en el ámbito nacional pueden señalarse varias etapas en las que el crecimiento del peso relativo del empleo público ha mostrado distinta intensidad y que ponen de manifiesto el carácter cíclico del empleo en general y, en menor medida, del empleo público ${ }^{3}$.

\section{$-1987.1991$}

La recuperación económica de este período se corresponde con una importante mejora del empleo asalariado del sector privado tanto en Castilla y León como en España. El número de asalariados del sector privado crece en 833.000 personas en España de las cuales 39.000 corresponden a Castilla y León. En este mismo período se crean 362.000 empleos públicos de los que 23.000 se crean en Castilla y León.

Tanto en el ámbito nacional como-en el regional es más fuerte el aumento del empleo público que el del empleo en general, por lo que el peso relativo de los empleados públicos aumenta en la economía nacional y en la regional (un punto porcentual en ambos casos).

$$
\text { - 1992-1994 }
$$

La ralentización del crecimiento económico propicia una disminución del empleo asalariado en el sector privado de la economía española y castellano-leonesa. Se destruyen 513.000 empleos asalariados en el sector privado de los que 19.500 corresponden al sector privado de la economía de Castilla y León.

En los dos primeros años de este período la reducción relativa del empleo del sector público es sensiblemente inferior a la reducción que experimenta el empleo asalariado del sector privado, lo que lleva a un aumento del peso relativo del primero de ellos tanto en la economía nacional como en la regional. No obstante, la importante reducción del empleo público que se produce durante el año 1994 en ambos ámbitos geográficos lleva a que el peso relativo de éste se mantenga en los niveles alcanzados en el año 1991. 


\section{Grabige Bol}

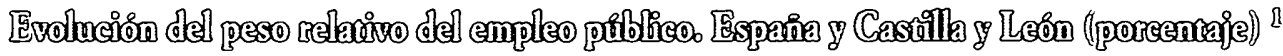

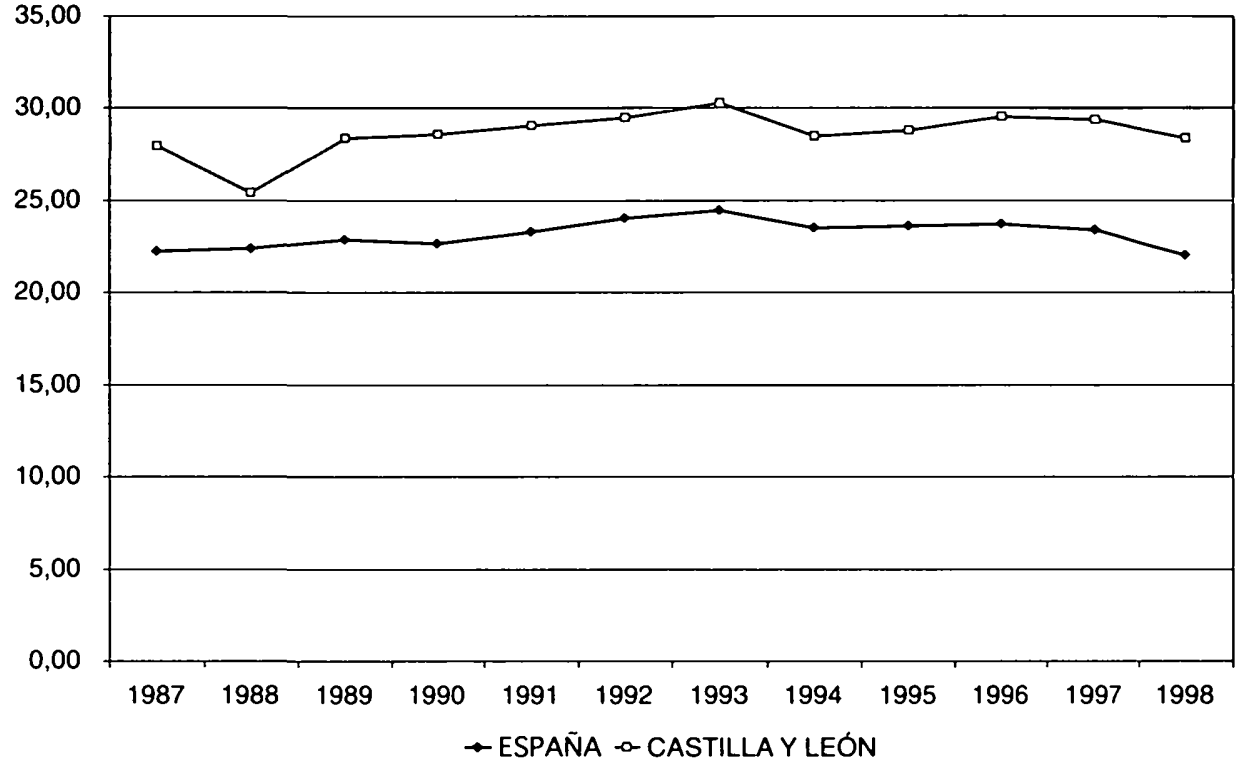

' El peso relativo del empleo público tanto en España como en Casulla y León se define como el cociente entre el número de asalariados públicos y el número de asalariados totales correspondientes a cada uno de los dos ámbitos geográficos.

Fuente: Elaborado a partir de los datos de la EPA

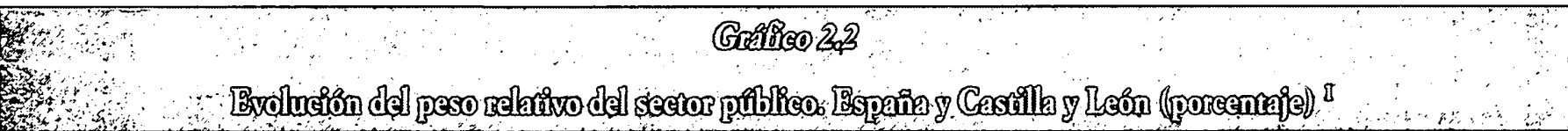

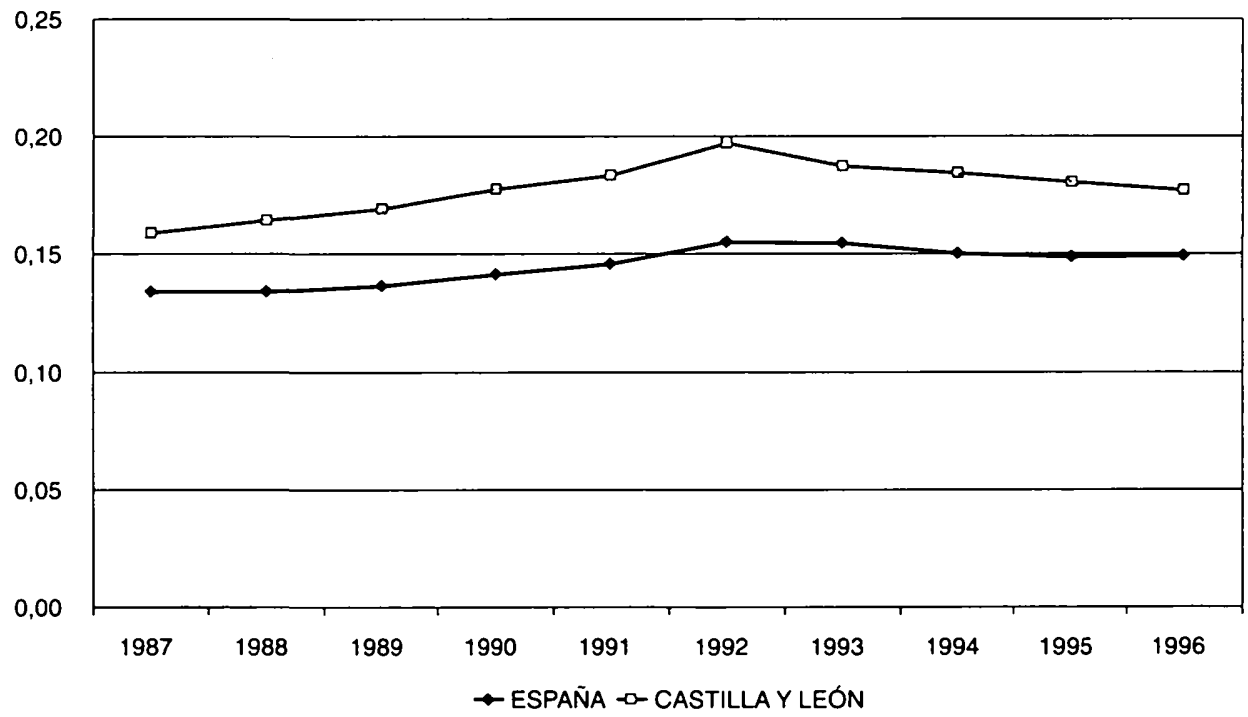

${ }^{1}$ El peso relativo del empleo público tanto en España como en Castilla y León se define como el cociente entre el valor añadido del sector servicios no destinados a la venta (servicios generales de las Administraciones públicas, Investigación y ensenanza no destinada a la venta, Sanidad no destinada a la venta y otros servicios no destinados a la venta) y el valor total generado en cada uno de los dos ámbitos geográficos. La información pública disponible no ha permitido obtener los datos correspondientes a los años 1997 y 1998.

Fuente: Elaborado a pariir de los datos de la Contabilidad Nacional de España y de la Contabilidad Regional de España. 


\section{$-1995.1998$}

La recuperación económica que se inicia en 1994 propicia un aumento de 1.416 .000 empleos asalariados en España de los que 182.000 (un 13\%) corresponden al sector público. En Castilla y León de los 43.500 empleos asalariados que se crean en estos cuatro años, 11.700 (un 27\%) son empleos públicos. El peso relativo del empleo público aumenta en la región y en 1998 se acentúa la diferencia con respecto al peso relativo que tiene en el conjunto del país, situándose en más de seis puntos porcentuales.

\section{Variaciones en la distribución del empleo público por sectores de la Administración}

En 1987, el empleo en el sector público de Castilla y León se distribuye de la siguiente forma: Administración Central, 41\%; Empresa e Institución Pública, 16\%; Administraciones de la Seguridad Social, 15\%; Corporaciones Locales, 15\% y Administración de la Comunidad Autónoma $12 \%{ }^{4}$.

Si comparamos esta distribución con la existente en el conjunto del país cabe señalar dos notas (Gráfico 3.1):
- Por un lado, el mayor peso que los asalariados de la Administración Central tienen en Castilla y León (a nivel nacional, los asalariados de la Administración Central representan solamente el 30\% del total de asalariados del sector público).

- Por otro lado, el menor peso relativo del empleo público de la Administración de la Comunidad Autónoma de Castilla y León. En España, el peso relativo del empleo público de las Administraciones de las Comunidades Autónomas sobre el total de asalariados públicos se sitúa en el $20 \%$. En otros términos, mientras que en el conjunto de España los asalariados públicos de las Administraciones de las Comunidades Autónomas representan, por término medio, el $4,5 \%$ del total de asalariados, en Castilla y León solamente representan el 3,4\%.

En cuanto al resto de Administraciones Públicas, el peso relativo de los asalariados de las Administraciones de la Seguridad Social es ligeramente superior en Castilla y León que en España y ligeramente inferior en el caso de las Corporaciones Locales y de las Empresas e Instituciones Públicas.

Entre 1987 y 1998, se produce un cambio en la distribución de los asalariados del sector público de Castilla y León a favor de las Administraciones de la Seguridad Social, de la Administración de la Comunidad Autónoma y de las Administraciones Locales y en contra de la Administración Central y de las Empresas e Instituciones Públicas; no obstante, el peso relativo del empleo público de la Administración de la Comunidad Autónoma de Castilla y León sigue estando por debajo del peso relativo del empleo público de las Administraciones de las Comunidades Autónomas en el conjunto de España (en 1998 la diferencia se sitúa en 12 puntos porcentuales).

En 1998, los porcentajes respectivos son: Administración Central, 38\%; Administraciones de la Seguridad Social, 20\%;

\section{Gráfico 3.1}

\section{Distribución de los empleados públicos según sectores de la Administración. Cástilla y León. 1987}

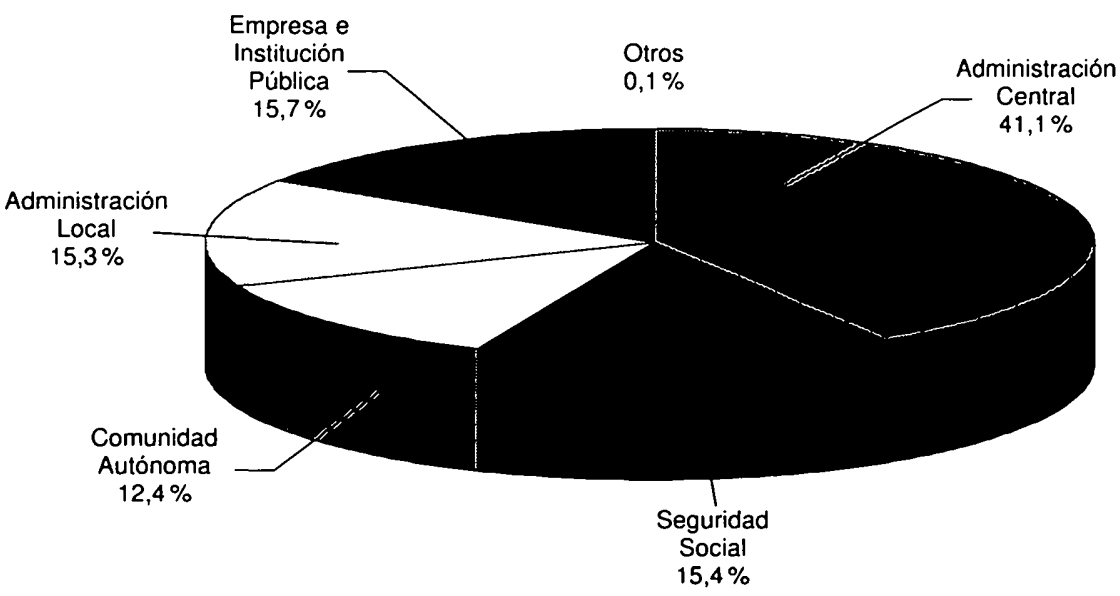




\section{Gratiog 332}

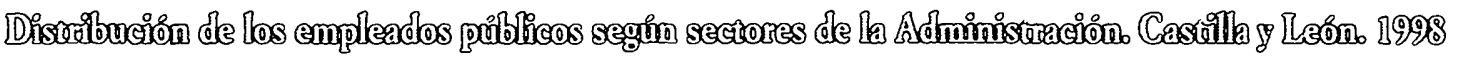

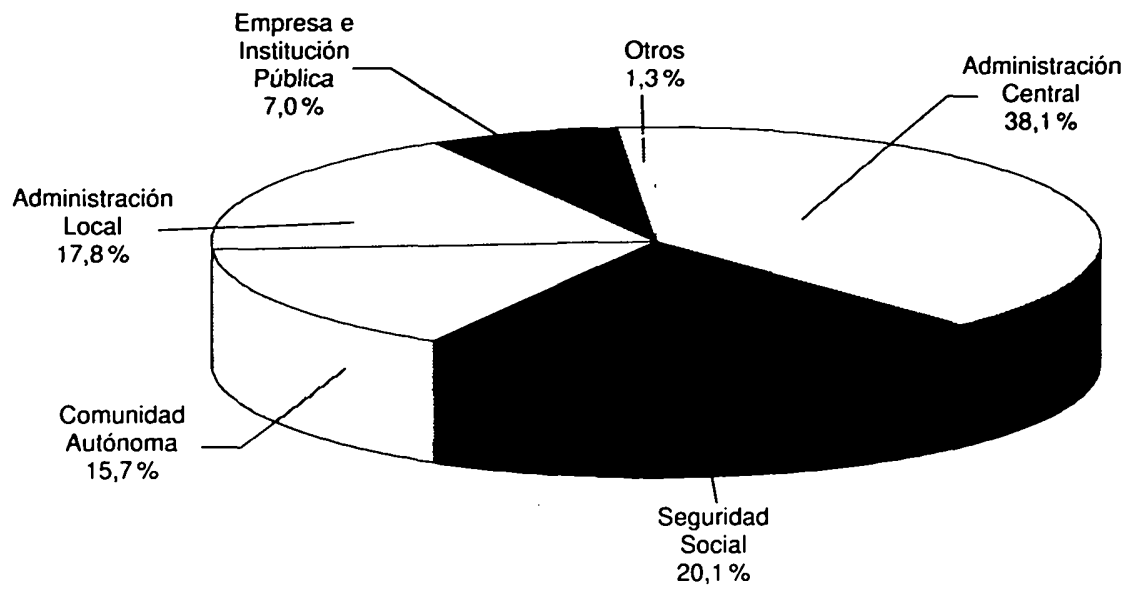

Fuente: Elaborado a partir de los datos de la EPA.

Corporaciones Locales, 18\%; Administración de la Comunidad Autónoma, 16\%, y Empresa e Institución Pública, 7\% (Gráfico 3.2).

Claramente se aprecia un rasgo diferencial respecto al cambio en la distribución ocurrido en el conjunto del país (Gráficos 3.1, 3.2, 3.3 y 3.4). Mientras que en España la disminución del peso relativo de los efectivos de la Administración Central y de las Empresas e Instituciones Públicas se traduce en un aumento del peso relativo de los asalariados de las Administraciones de las Comunidades Autónomas y, en menor medida, de los asalariados de las Administraciones de la Seguridad Social, en Castilla y León se traduce fundamentalmente en un aumento del peso relativo de los asalariados de las Administraciones de la Seguridad Social y, en menor medida de los asalariados de la Administración de la Comunidad Autónoma y de las Corporaciones Locales 5 .

\section{Caracterización de los empleados públicos en Castilla y León}

La caracterización de los asalariados públicos en Castilla y León se va a realizar considerando ciertas características personales, como la edad, el sexo y el nivel de estudios, y ciertas características relacionadas con el trabajo desempeñado, como el nivel profesional, el tipo de contrato laboral y el sector de actividad económica.

El nivel de estudios se clasifica con cuatro niveles: analfabetos o sin estudios; estudios primarios, donde se incluyen los estudios de EGB, el bachillerato elemental y el certificado de escolaridad o equivalente; estudios medios, que incluyen formación profesional, BUP, artes aplicadas y similares, y estudios superiores que incluyen carreras universitarias de ciclo corto y largo, doctorado y cualquier otro tipo de estudios superiores.

La formación complementaria se entiende como la realiza. ción de algún tipo de estudio orientado a alguna actividad o profesión de duración superior a un año, bien sean estudios de postgrado, estudios realizados en empresas o centros especializados o estudios por correspondencia.

El nivel profesional se ha agrupado en tres escalones: puestos de dirección o similares; trabajadores cualificados tales como ayudantes técnicos sanitarios, especialistas y técnicos en contabilidad, profesionales de las fuerzas armadas, jefes de taller, etc., $y$, finalmente, trabajadores semicualificados y no cualificados.

Los contratos laborales se han agrupado en contratos temporales, que incluyen contratos de formación y aprendizaje, de temporada y otras modalidades similares, y contratos indefinidos, ya sean continuos o discontinuos.

Los sectores de actividad económica considerados son los tradicionales: primario, secundario y terciario ${ }^{6}$. 


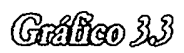

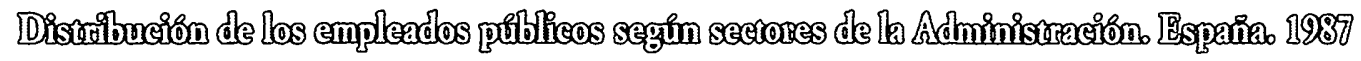

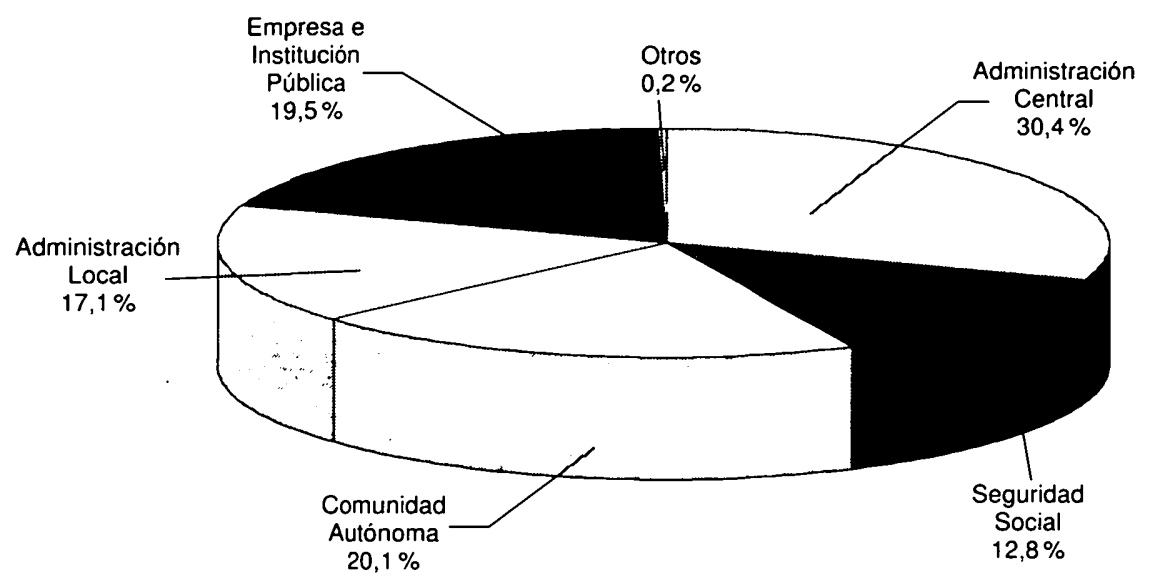

Fuente: Elaborado a partir de los datos de la EPA.
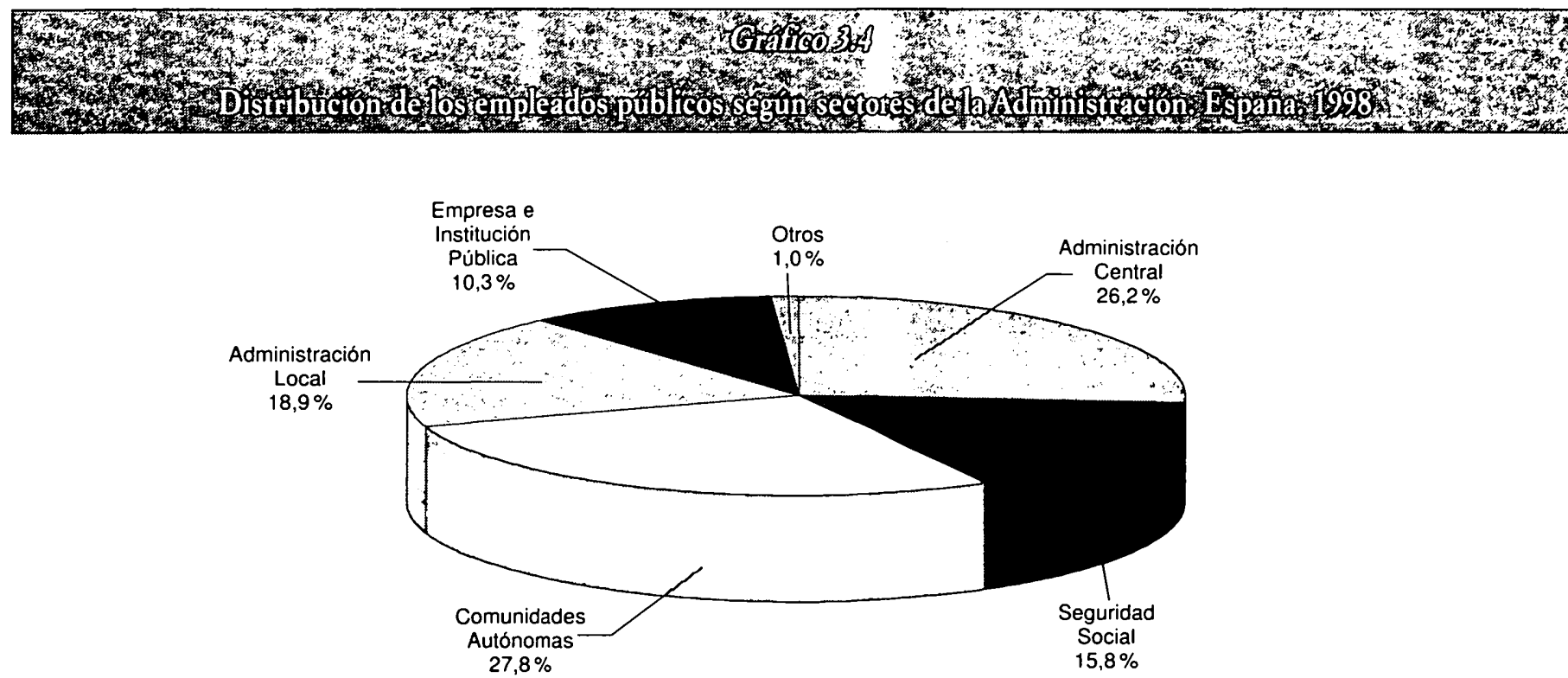

Fuente: Elaborado a partir de los datos de la EPA.

La distribución de los empleados públicos, según las categorías anteriores, ha variado entre los años 1987 y 1998, sin que se aprecien notables diferencias entre los asalariados públicos de Castilla y León y el conjunto de asalariados públicos de España (Tabla 4.1).

El peso de los empleados públicos de edad inferior a treinta y cinco años y de edad superior a cuarenta y cinco años ha disminuido en relación con el peso de los empleados públicos de edades entre treinta y cinco y cuarenta y cinco años ( $T a$ bla 4.1). En relación con el sexo, la participación de las mujeres ha aumentado en este período, aproximándose a la participación de los hombres (Tabla 4.1). Al considerar ambas características de forma conjunta se observa que la participación femenina es inferior a la masculina en 1987 con independencia del grupo de edad, pero en 1998 la participación de las mujeres supera a la participación de los hombres entre los asalariados públicos de menor edad (Tabla 4.2).

El nivel de estudios también ha variado notablemente, y así en 1987 predominan los empleados públicos con estudios primarios, mientras que en 1998 predominan los empleados 


\begin{tabular}{|c|c|c|c|c|}
\hline Porseman & 2018 & poote & & \\
\hline & Castil & León & & \\
\hline & 1987 & 1998 & 1987 & 1998 \\
\hline 35 años 0 mar?or & $35 \times$ & $2 \leqslant 2$ & $4 !, 2$ & 30.5 \\
\hline Entre 35 y 45 años & 26,3 & 39,3 & 26,1 & 35,6 \\
\hline Majores de 45 años & 37,9 & 34,4 & $33, \overline{7}$ & 33,9 \\
\hline Hombres & 66,3 & 52,5 & 66,2 & 53,4 \\
\hline Mujeres & 33,7 & 47,3 & 33,8 & 46,6 \\
\hline Analfabetos o sin estudios & 1,1 & 0,6 & 4,7 & 2,0 \\
\hline Estudios primarios & 43,0 & 26,5 & 42,9 & 27,2 \\
\hline Estudios medios & 18,7 & 27,4 & 17,7 & 25,7 \\
\hline Estudios superiores & 37,1 & 45,4 & 34,7 & 45,1 \\
\hline Estudios complementarios & 4,9 & 9,9 & 5,5 & 10,2 \\
\hline Puestos de dirección & 25,8 & 38,6 & 25,0 & 34,4 \\
\hline Puestos cualificados & 17,3 & 16,9 & 16,8 & 17,1 \\
\hline Puestos semi y no cualificados & 56,9 & 44,5 & 58,2 & 48,5 \\
\hline Contrato indefinido & 92,7 & 83,7 & 91,5 & 82,2 \\
\hline Contrato temporal & 7,3 & 16,3 & 8,5 & 17,8 \\
\hline Sector primario & 5,7 & 2,1 & 4,7 & 1,9 \\
\hline Sector secundario & 5,1 & 2,9 & 9,0 & 3,8 \\
\hline Sector terciario & 89,2 & 95,0 & 86,3 & 94,3 \\
\hline
\end{tabular}

Fuente: Encuesta de Población Activa, $2 .^{\circ}$ trimestre, 1987 y 1998. Elaboración propia.

públicos con educación superior (Tabla 4.1). Además, mientras que en 1987 el peso de los asalariados públicos con estudios primarios es importante en todos los grupos de edad, en 1998 se localiza en el grupo de mayor edad donde el porcentaje de asalariados públicos con estudios primarios se aproxima considerablemente al porcentaje de asalariados con estudios superiores, y, por el contrario, en el grupo más joven predominan los empleados públicos con estudios superiores (Tabla 4.3).

Al relacionar el nivel de estudios y el sexo de los asalariados públicos en 1987 se aprecia por una parte un mayor peso de los asalariados públicos con estudios primarios entre los varones y por otra parte, un mayor peso de los asalariados públicos

\begin{tabular}{|c|c|c|c|c|c|c|c|}
\hline & & & & & & & \\
\hline Pore & ace & 5 de $x$ & miacedo & porbliec & 8 segute & gdted? & ह्रा० \\
\hline & & Menor o iq & al 35 años & Entre 36 & 45 años & Más de & 5 años \\
\hline & & Hombre & Mujer & Hombre & Mujer & Hombre & Mujer \\
\hline Castilla & $198 \overline{1}$ & 59.01 & 40,99 & 61.74 & 38,26 & 76.22 & 23,78 \\
\hline & 1998 & 43,53 & 56,47 & 52,57 & 47.43 & 59,19 & 40,81 \\
\hline$F_{8}$ & 1987 & 56,84 & 43,16 & 66,55 & 33,45 & 77,26 & 22,74 \\
\hline Lspana & 1998 & 48,14 & 51,86 & 51,15 & 48,85 & 60,54 & 39,46 \\
\hline
\end{tabular}

Fuente: Encuesta de Población Activa, $2^{\circ}{ }^{\circ}$ trimestre, 1987 y 1998 . Elaboración propia.

con estudios superiores entre las mujeres. En 1998 se mantiene esa diferencia educativa en el caso de las mujeres, mientras que para los hombres el peso de los asalariados públicos con estudios primarios se reduce considerablemente a favor de los estudios medios y superiores (Tabla 4.4).

\section{पृab 483}

\begin{tabular}{|c|c|c|c|c|c|c|c|c|c|}
\hline & & \multicolumn{4}{|c|}{ Hombre } & \multicolumn{4}{|c|}{ Mujer } \\
\hline & & Analf. & Prim. & Med. & Sup. & Analf. & Prim. & Med. & Sup. \\
\hline \multirow{2}{*}{$\begin{array}{l}\text { Castilla } \\
\text { y León }\end{array}$} & 1987 & 1,20 & 51,47 & 17,60 & 29,73 & 1,05 & 26,44 & 20,94 & 51,57 \\
\hline & 1998 & 0,56 & 34,06 & 27,57 & 37,81 & 0,75 & 18,21 & 27,28 & 53,76 \\
\hline \multirow{2}{*}{ España } & 1987 & 5,16 & 50,44 & 17,09 & 27,31 & 3,81 & 28,12 & 18,94 & 49,13 \\
\hline & 1998 & 2,27 & 34,33 & 26,57 & 36,83 & 1,73 & 18,84 & 24,79 & 54,65 \\
\hline
\end{tabular}

Fuente: Encuesta de Población Activa, $2{ }^{\circ}$ trimestre, 1987 y 1998. Elaboración propia.

A pesar de la mayor cualificación poseída por los asalariados públicos a lo largo de todo este tiempo, la formación complementaria continúa siendo escasa (Tabla 4.1). En 1987 este tipo de formación se concentra en el grupo más joven (hasta treinta y cinco años), mientras que en 1998 llega en la misma

\section{Tab? 433}

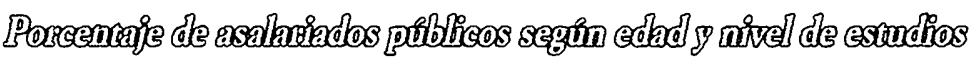

\begin{tabular}{|c|c|c|c|c|c|c|c|c|c|c|c|c|c|}
\hline & & \multicolumn{4}{|c|}{ Menor o igual 35 anios } & \multicolumn{4}{|c|}{ Entre 36 y 45 años } & \multicolumn{4}{|c|}{ Más de 45 años } \\
\hline \multirow{2}{*}{$\begin{array}{l}\text { Castilla } \\
\text { y León }\end{array}$} & 1987 & 0,49 & 30,37 & 34,57 & 34,57 & 1,01 & 40,27 & 13,09 & 45,64 & 1,86 & 56,88 & 7,69 & 33,57 \\
\hline & 1998 & 0,34 & 19,10 & 34,33 & 46,23 & 0,28 & 20,00 & 34,36 & 45,36 & 1,31 & 39,64 & 14,28 & 44,77 \\
\hline España & 1987 & 0,94 & 30,57 & 28,86 & 39,64 & 2,98 & 45,76 & 13,80 & 37,45 & 10,55 & 55,44 & 7,42 & 26,60 \\
\hline
\end{tabular}

Fuente: Encuesta de Población Activa, 2. ${ }^{\circ}$ trimestre, 1987 y 1998. Elaboración propia. 


\begin{tabular}{|c|c|c|c|c|}
\hline \multicolumn{5}{|c|}{$\begin{array}{c}\text { Tabla } 4.5 \\
\text { Porcentaje de asalariados públicos con estudios } \\
\text { complementarios según edad }\end{array}$} \\
\hline & & Menor 0 igual 35 años & Entre 36 y 45 años & Más de 45 años \\
\hline \multirow{2}{*}{$\begin{array}{l}\text { Castilla } \\
\text { y León }\end{array}$} & 1987 & 9,14 & 4,36 & 1,17 \\
\hline & 1998 & 9,48 & 10,54 & 9,48 \\
\hline \multirow{2}{*}{ España } & 1987 & 9,48 & 4,10 & 0,78 \\
\hline & 1998 & 9,78 & 10,10 & 9,28 \\
\hline
\end{tabular}

Fuente: Encuesta de Población Activa, 2. ${ }^{\circ}$ trimestre, 1987 y 1998 . Elaboración propia.

\begin{tabular}{|c|c|c|c|}
\hline \multicolumn{4}{|c|}{$\begin{array}{c}\text { Tabla } 4.6 \\
\text { Porcentaje de asalariados públicos } \\
\text { con estudios complementarios según sexo }\end{array}$} \\
\hline & & Hombre & Mujer \\
\hline \multirow{2}{*}{$\begin{array}{l}\text { Castilla } \\
\text { y León }\end{array}$} & 1987 & 2,39 & 3,48 \\
\hline & 1998 & 8,59 & 11,34 \\
\hline \multirow{2}{*}{ España } & 1987 & 4,50 & 7,40 \\
\hline & 1998 & 9,68 & 10,85 \\
\hline
\end{tabular}

Fuente: Encuesta de Población Activa, 2. ${ }^{\circ}$ trimestre, 1987 y 1998. Elaboración propia. medida a todos los grupos de edad, sin observarse importantes diferencias entre sexos (Tablas 4.5 y 4.6).

La categoría profesional predominante en ambos años entre los asalariados públicos de Castilla y León es la categoría de trabajadores semicualificados o no cualificados, aunque en 1998 se detecta un notable aumento del empleo en los puestos de dirección (Tabla 4.1). La mayoría de los asalariados públicos que carecen de estudios ocupan puestos no cualificados, mientras que los asalariados públicos con estudios universitarios desempeñan, en mayor proporción, trabajos de dirección; esta pauta se mantiene en ambos años (Tabla 4.7). Se observa que en 1987 las diferencias entre sexos son mayores en los puestos de trabajo de menor categoría, mientras que en 1998 la mayor diferencia se observa en los puestos de trabajo cualificado. También destaca en 1998 la mayor presencia de mujeres, superando a los hombres, en los puestos de dirección (Tabla 4.8).

Los contratos predominantes son los de carácter indefinido aunque los contratos de carácter temporal han cobrado importancia en el último año (Tabla 4.1). Mientras que los contratos indefinidos predominan en los hombres, los contratos temporales han pasado de estar concentrados en los hombres a ser más frecuentes en las mujeres (Tabla 4.9). Asimismo, cabe destacar que los contratos indefinidos se concentran en el sector terciario (servicios) mientras que los contratos temporales han

\begin{tabular}{|c|c|c|c|c|c|c|c|c|c|c|c|c|c|}
\hline \multicolumn{14}{|c|}{$\because \cdots$} \\
\hline \multicolumn{14}{|c|}{ Porcentaje de asalariados públicos según nivel educativo y categoria socioeconómica } \\
\hline & & \multicolumn{3}{|c|}{ Analfabetos } & \multicolumn{3}{|c|}{ Est. Primarios } & \multicolumn{3}{|c|}{ Est. Medios } & \multicolumn{3}{|c|}{ Est. Superiores } \\
\hline & & Direc. & Cualf. & No cualf. & Direc. & Cualf. & No cualf. & Direc. & Cualf. & No cualf. & Direc. & Cualf. & No cualf. \\
\hline \multirow{2}{*}{$\begin{array}{l}\text { Castilla } \\
\text { y León }\end{array}$} & 1987 & 0,00 & 0,00 & 100,0 & 1,44 & 13,17 & 85,39 & 7,08 & 20,75 & 72,17 & 64,73 & 21,01 & 14,25 \\
\hline & 1998 & 10,73 & 0,00 & 89,27 & 27,46 & 19,60 & 52,94 & 39,67 & 17,30 & 43,03 & 55,08 & 12,82 & 32,10 \\
\hline \multirow{2}{*}{ Espania } & 1987 & $0,4 i$ & 2,83 & 96,70 & 1,24 & 13,69 & 85,07 & 8,40 & 18,55 & 73,06 & 66,42 & 21,77 & 11,81 \\
\hline & 1998 & 14,66 & 4,31 & 81,03 & 23,92 & 17,86 & 58,22 & 37,31 & 17,12 & 45,57 & 50,00 & 17,30 & 32,70 \\
\hline
\end{tabular}

Fuente: Encuesta de Población Activa, 2. ${ }^{\circ}$ trimestre, 1987 y 1998. Elaboración propia.

\section{Tabla 4.8}

Porcentaje de asalariados públicos según categoría socioeconómica y sexo

\begin{tabular}{|c|c|c|c|c|c|c|c|}
\hline & & \multicolumn{2}{|c|}{ Dirección } & \multicolumn{2}{|c|}{ Cualificados } & \multicolumn{2}{|c|}{ Semi o no cualificados } \\
\hline \multirow{2}{*}{ Castilla y León } & 1987 & 54,14 & 45,86 & 63,08 & 36,92 & 72,34 & 27,66 \\
\hline & 1998 & 41,63 & 58,37 & 83,65 & 16,35 & 57,82 & 42,18 \\
\hline España & 1987 & 56,99 & 43,01 & 56,77 & 43,23 & 72,91 & 27,09 \\
\hline
\end{tabular}

Fuente: Encuesta de Población Activa, 2. ${ }^{\circ}$ trimestre, 1987 y 1998. Elaboración propia. 


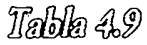

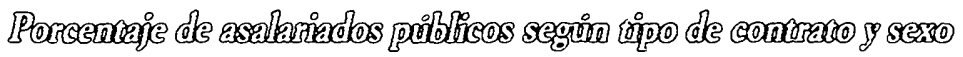

\begin{tabular}{|c|c|c|c|c|c|}
\hline & & \multicolumn{2}{|c|}{ Contrato indefinido } & \multicolumn{2}{|c|}{ Contrato temporal } \\
\hline & & Hombre & Mujer & Hombre & Mujer \\
\hline Castilla y León & 1987 & 6,16 & 33.84 & $6 \bar{i} .4 \bar{j}$ & 32.53 \\
\hline \multirow{2}{*}{ Espana } & 1987 & 66,73 & 33,27 & 61,02 & 38,98 \\
\hline & 1998 & 55,76 & 44,24 & 42,53 & 57,47 \\
\hline
\end{tabular}

Fuente: Encuesta de Población Activa, $2 .^{\circ}$ trimestre, 1987 y 1998. Elaboración propia.

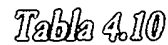

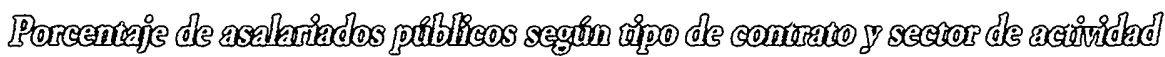

\begin{tabular}{|c|c|c|c|c|c|c|c|}
\hline & & \multicolumn{3}{|c|}{ Contrato indefinido } & \multicolumn{3}{|c|}{ Contrato temporal } \\
\hline \multirow{2}{*}{ Castilla y León } & 1987 & 4,0 & 5,05 & 90,94 & 27,71 & 6,02 & 66,27 \\
\hline & 1998 & 2,17 & 3,06 & 94,77 & 1,50 & 1,88 & 96,62 \\
\hline \multirow{2}{*}{ España } & 1987 & 4,30 & 8,85 & 86,84 & 8,27 & 11,02 & 80,71 \\
\hline & 1998 & 1,73 & 3,48 & 94,79 & 2,68 & 5,45 & 91,87 \\
\hline
\end{tabular}

Fuente: Encuesta de Población Activa, 2. ${ }^{\circ}$ trimestre, 1987 y 1998 . Elaboración propia.

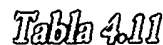

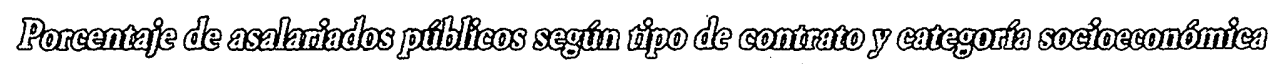

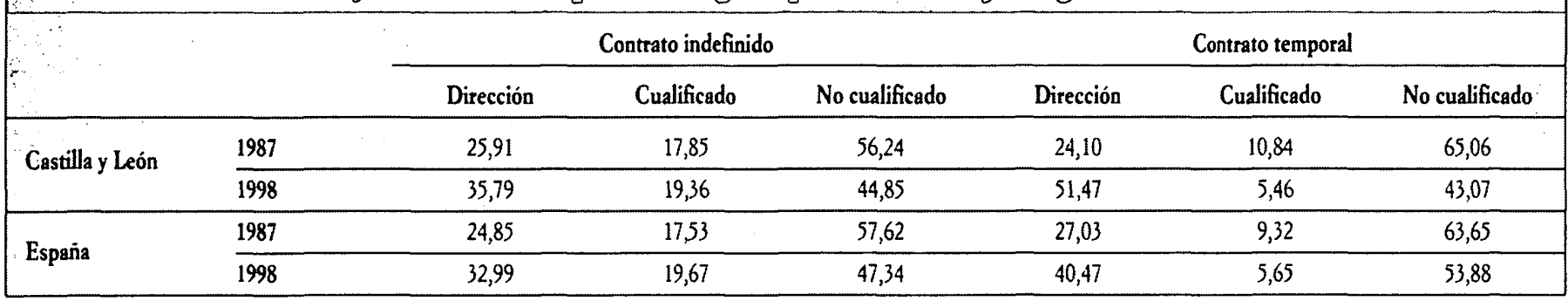

Fuente: Encuesta de Población Activa, 2. ${ }^{\circ}$ trimestre, 1987 y 1998. Elaboración propia.

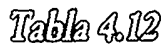

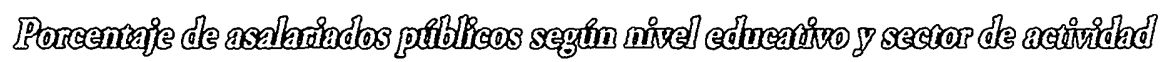

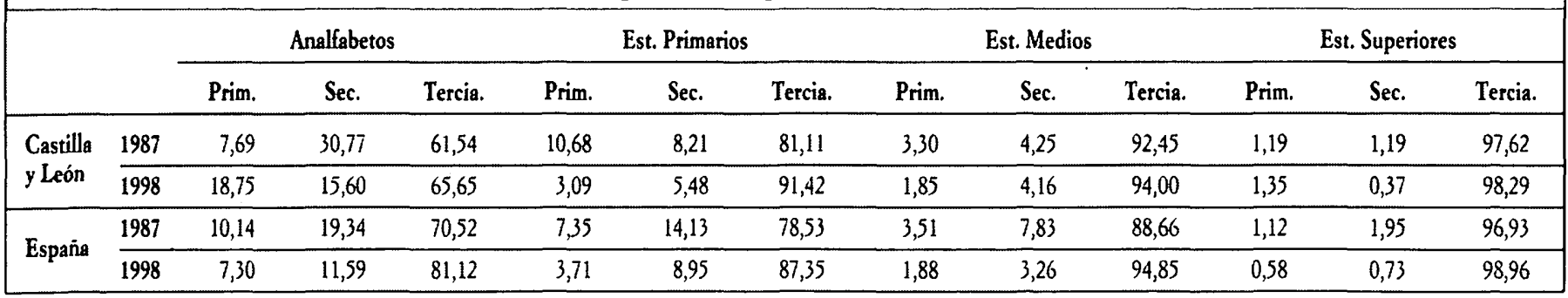

Fuente: Encuesta de Población Activa, 2. ${ }^{\circ}$ trimestre, 1987 y 1998. Elaboración propia. 
pasado de tener en Castilla y León relativa importancia en el sector primario (agrario) a concentrarse únicamente en el sector terciario (Tabla 4.10). En 1987 ambos tipos de contratos dominaban entre los puestos no cualificados, sin embargo, el aumento en el número de asalariados que desempeña un puesto de dirección ha extendido la contratación temporal e indefinida hasta esta categoría (Tabla 4.11).

Finalmente, destacar que una amplia mayoría del empleo público se localiza en el sector terciario, siendo cada vez mayor el peso de éste en detrimento tanto del sector primario como del sector secundario (Tabla 4.1), con independencia del nivel educativo poseído (Tabla 4.12).

\section{Conclusiones}

Los rasgos más sobresalientes de la estructura del empleo público en Castilla y León, según los resultados de este trabajo, son los siguientes:

En primer lugar, destaca el porcentaje de asalariados públicos en Castilla y León, que es ligeramente superior al del resto del país (aproximadamente un 5\% más). Este resultado se corresponde con el mayor peso relativo que el sector público de Castilla y León tiene en el valor añadido total que se genera en la región. También es destacable el hecho de que la Administración de la Comunidad Autónoma castellano-leonesa emplea un porcentaje de personas inferior al conjunto de las Administraciones Autonómicas del país; no obstante, debe tenerse en cuenta que esto puede ser consecuencia de que el proceso de transferencia de competencias desde la Administración Central no se ha producido ni en el mismo momento ni con la misma intensidad en las diferentes Comunidades Autónomas.

En segundo lugar, y en relación con las características de los empleados públicos, cabe señalar que no se aprecian diferencias entre lo observado para Castilla y León y lo observado para el conjunto de España. Y así, destaca la mayor participación de la mujer entre los empleados públicos de menor edad; la mayor cualificación, especialmente entre los asalariados públicos mujeres, predominando los estudios superiores; la escasa formación complementaria; el aumento en la Administración Pública de los puestos de dirección, con mayor presencia de la mujer en los mismos; el aumento de la contratación laboral, que afecta en mayor medida a las mujeres, a pesar de que predomina la contratación indefinida, y, por último, la práctica terciarización del empleo público a lo largo de todo este tiempo.

\footnotetext{
* Profesores Asociados. Universidad de Valladolid.

${ }^{1}$ Cabe señalar, sin embargo, tres comunicaciones presentadas en el $6 .^{\circ}$ Congreso de Economía Regional de Castilla y León: Carrrascal, Martin Román y Pérez Dominguez (1998), Mate Garcia y Pérez Dominguez (1998) y Rodriguez Caba. LLeRo y Valiente Garcia (1998).

2 Debe señalarse que, a pesar de ser la EPA la base de datos que incorpora la información necesaria para realizar este trabajo, su representatividad se ve reducida en el análisis de tipo regional o de carácter especifico.

${ }^{3}$ No hay que olvidar que el empleo público está asociado a los servicios públicos $y$, por tanto, responde a factores distintos que otros sectores. Por otro lado, el empleo público, como se señala posteriormente, es fundamentalmente indefinido $y$, por tanto, con grandes rigideces para ajustarse a la baja.

${ }^{4}$ En la categoría Empresa e Institución Pública se incluyen las personas que trabajan en empresas financieras y no financieras controladas por el sector público sin distinguir el nivel de gobierno del que dependen (central, autonómico o local).
}

\begin{abstract}
' Nótese que se está comparando la distribución de los empleados públicos de Castilla y León y los cambios producidos en la misma entre 1987 y 1998 con la distribución y los cambios registrados en el mismo periodo en el conjunto del pais, por término medio. Resulta obvio que la desigual distribución de competencias entre Comunidades Autónomas en el período inicial y la diferente evolución en la transferencia de competencias a las distintas Comunidades Autónomas a lo largo del período considerado está detrás de las diferencias registradas en el periodo inicial y de los cambios detectados. En otros términos, los resultados serían distintos si la comparación se hiciera con aquellas Comunidades en las que las competencias iniciales y el proceso de transferencia de competencias fueran similares a los registrados en Castilla y León.

- Si bien la mayoria del empleo público se localiza en el sector terciario (servicios) se aprecia la existencia de empleo público también en el sector primario (extracción mineral, actividades forestales, etc.) y en el sector secundario (siderurgia, producción y primera transformación de metales, obras públicas, etc.).
\end{abstract}

\section{Bibliografía}

Carrascal, U.; Martin Romín, A. L., y Pérez Dominguez, C. (1998), «El perfil más probable del empleado público castellano-leonés», $6{ }^{\circ}$ Congreso de Economía Regional de Castilla y León. Comunicaciones I: 429-441.
Carrasco, C. G., y Dominguez Martinez, J. M. (1995), «El crecimiento del sector público en España y en los países de la OCDE desde la perspectiva del empleo público», Presupuesto y Gasto Público, núm. 15: 29.64. 
Doninguez Martivez, J. M. (1989-1990), «El empleo público en los países occidentales desarrollados: aspectos teóricos y empíricos», Cuadernos de Ciencias Eco nómicas y Emprexariales, núm. 20: 73-113.

Garcia López, J., y ÂlviRA Martis, F. (1996), «Personal al servicio de las Administraciones Públicas», Papeles de Economía Española, núm. 68: 199-206

Mate Garcia, J. J., y Pérez Dominguez, C. (1998), «El empleo público en Castilla y León según los datos del censo de población de 1991 », $6{ }^{\circ}$ Congreso de Economia Regional de Castilla y León. Comunicaciones I: 290-304.
Poıo ANDrés, C. (1992), «El empleo en la economía española: 1976-1992», Ekonomiaz, núm. 24: 72-103.

Rodriguez Cabaluero, J. C., y Valiente Garcia, A (1998), «Evolución y caracterización del empleo público en Castilla y León: un análisis comparado para el periodo 1987-1996», 6. ${ }^{\circ}$ Congreso de Economia Regional de Castilla y León. Comunicaciones I; 488-505. 
